diz hierfür lässt sich die extrem niedrige Zahl der Plattformnutzer lesen, die nur ein oder zwei E-Petitionen mitzeichneten und danach die Plattform nicht mehr verwendeten.

Wenn also der „Aufstand der Netzbürger“ 47 auf das gelassene „wir sollten vielleicht chatten" 48 der gewählten Volksvertreter trifft, ist unklar, welche gestalterische Form der vorher geweckte Mitbestimmungsanspruch erhält. Vielleicht steht exemplarisch für dieses Gefühl eine Äußerung des Twitter-Nutzers Max Winde: „Ihr werdet euch noch wünschen, wir wären politikverdrossen." ${ }^{49}$

47 Marcel Rosenbach / Hilmar Schmundt, Aufstand der Netzbürger, in: Der Spiegel vom 3. August 2009, S. $26-28$.

48 Ole Reißmann, Vielleicht sollten wir chatten, in: Spiegel Online vom 22. Februar 2010, http:// www.spiegel.de/netzwelt/netzpolitik/0,1518,679582,00.html (Abruf am 18. Februar 2011).

49 MaxWinde (@343max), Ihr werdet euch noch wünschen wir wären politikverdrossen, 18. Juni 2009, http://twitter.com/343max/status/2228357957 (Abruf am 18. Februar 2011).

\title{
Noch immer „Stiefkind der Sozialwissenschaften“? Ein Plädoyer für mehr politikwissenschaftliche Forschung über Rechnungshöfe*
}

\author{
Markus Seyfried
}

Es gibt nur wenige Institutionen, die von den Sozialwissenschaften so konsequent ignoriert werden wie die Rechnungshöfe. Dies gilt im deutschen Fall umso mehr für die Landesrechnungshöfe. Auf den ersten Blick mag dies verwundern, da die Schuldenlast der öffentlichen Haushalte immer mehr zunimmt und die Bedeutung der Finanzkontrolleure als kritische „Generalisten"1 steigen müsste. Auf den zweiten Blick jedoch spiegelt sich in dem wissenschaftlichen Desinteresse das generelle Schattendasein wider, das die Rechnungshöfe fristen. Die Institutionen der Finanzkontrolle werden vom Bürger weitgehend ignoriert - abgesehen von einigen wenigen medienwirksamen Skandalen - und von der Politik mitunter milde belächelt.

Die geringe Beachtung der Rechnungshöfe - ähnlich wie von Nils Diederich u.a. in Bezug auf den Bundesrechnungshof festgestell $\mathrm{t}^{2}$ - verwundert insofern, als gerade die Sozialwissenschaften dafür prädestiniert sind, sich an schwer greifbare Untersuchungsgegenstän-

* Für sehr hilfreiche Anmerkungen und Kritik danke ich Julia Fleischer, Sylvia Veit und Bastian Jantz sowie der ZParl-Redaktion.

1 Vgl. Werner Jann / Kai Wegrich, Wie bürokratisch ist Deutschland? Und warum? Generalisten und Spezialisten im Entbürokratisierungsspiel, in: Der moderne Staat, 1. Jg. (2008), H. 1, S. 49 - 72; Hans Herbert von Arnim, Gemeinwohl und Gruppeninteressen. Die Durchsetzungsschwäche allgemeiner Interessen in der pluralistischen Demokratie, Frankfurt am Main 1977, S. 373; ders., Wirksamere Finanzkontrolle bei Bund, Ländern und Gemeinden. Analyse und Reformvorschläge, Karl-Bräuer Institut des Bundes der Steuerzahler, H. 42, Bad Wörishofen 1978, S. 54.

2 Vgl. Nils Diederich / Franz-Otto Gilles / Gerhard Otto / Gundolf Otto / Rainer Weinert, Die Institution Rechnungshof, Stiefkind der Sozialwissenschaften, in: ZParl, 15. Jg. (1984), H. 4, S. $479-$ 494, S. 493 f. 
de heranzuwagen. Dabei gibt es eine Vielzahl unterschiedlichster Forschungsfragen, die empirisch noch nahezu unbeleuchtet sind. Wie unabhängig sind beispielsweise die Rechnungshöfe tatsächlich? Was sind die zentralen Determinanten ihrer Wirksamkeit? Wie funktioniert das Zusammenspiel mit den Haushaltsausschüssen und dem Parlament? Fungiert der Rechnungshof als "parlamentarischer Hilfsdienst“" rene „vierte Gewalt“ “? Ist die These vom Präventiveffekt durch drohende Prüfung der Rechungshöfe tatsächlich haltbar? In welchen Bereichen hat sich die Rolle der Rechnungshöfe verändert und warum?

Generell gibt es hierzu bisher kaum zusammenhängende oder gar vergleichende Datenerhebungen. Nur vereinzelt kursieren Zahlen und Fakten, die je nach Bundesland ganz unterschiedlich aufgearbeitet sind. Darüber hinaus fehlt es an strukturierten Forschungsansätzen für eine systematische Auswertung von Jahresberichten, Erfolgsberichten, Pressemitteilungen, Plenarprotokollen, Beschlussempfehlungen der Haushaltsausschüsse oder Stellungnahmen der Exekutive zu den Prüfberichten der Rechnungshöfe. Nahezu alle offiziellen Dokumente werden bisher nur zur Illustration, nicht aber für eine systematische Erhebung oder gar Analyse verwendet. Auch die Innensicht der Rechnungshöfe ist - soweit nicht in einzelnen Buchbeiträgen oder Jubiläumsschriften bearbeitet ${ }^{5}$ - nahezu unbekannt. Dabei würde sich dieses Feld besonders für Befragungen und Interviews eignen, weil hier sehr gut zwischen Prüfer, Geprüftem und Adressaten des Rechnungshofberichtes kontrastiert werden könnte.

\section{Probleme in der Forschung über Rechnungshöfe}

Ausgangspunkt der folgenden Betrachtungen ist die bereits erwähnte Untersuchung von Diederich u.a., die den Stiefkind-Status der Rechnungshöfe in den Sozialwissenschaften erstmals kritisiert haben. Die Ergebnisse ihres Beitrages lassen sich im Wesentlichen auf folgende zentrale Argumente komprimieren: (1) Es gibt keinen beziehungsweise nur einen sehr geringen kumulativen Erkenntnisgewinn in den wissenschaftlichen Diskussionen über die Rechnungshöfe. (2) Es kursieren zahlreiche Thesen und Positionen über Rechnungshöfe und Finanzkontrolle, zu denen nur in Ausnahmefällen empirisch fundierte Beweisführungen erbracht wurden. (3) Wo diese vorliegen, erweisen sie sich oftmals als mangelhaft und selektiv. (4) Die verwendeten normativen Ansätze stoßen regelmäßig an ihre Grenzen, wenn es darum geht, das Wesen der Rechnungshöfe zu erfassen und zu erklären. ${ }^{6}$

3 Vgl. kritisch dazu Alois Igelspacher, Die staatliche Finanzkontrolle in Bayern, München 2002, S. 76.

4 Mortimer A. Dittenhofer, Is Auditing a Fourth Power?, in: State Government, 43. Jg. (1970), H. 3, S. 179 - 183, S. 181.

5 Vgl. Rudolf Dieckmann, Standortbestimmung, Beträge zum 50. Jahrestag der Gründung eines unabhängigen Rechnungshofes der Freien und Hansestadt Hamburg, Hamburg 1998; Rechnungshof des Saarlandes, Finanzkontrolle im Saarland. 25 Jahre Rechnungshof des Saarlandes, Merzig 1988; Rechnungshof Nordrhein-Westfalen, 40 Jahre Landesrechnungshof NordrheinWestfalen 1948-1988, Düsseldorf 1988; Sächsischer Rechnungshof, Finanzkontrolle in Sachsen. Der Rechnungshof des Freistaates Sachsen, Leipzig 1995.

6 Vgl. Nils Diederich / Franz-Otto Gilles / Gerhard Otto / GundolfOtto / Rainer Weinert, a.a.O. (Fn. 2), S. $493 \mathrm{f}$. 
Diese zum Teil heftige Kritik an den Forschungsarbeiten über Rechnungshöfe wurde vor über 25 Jahren formuliert, und es ist zu fragen, ob sie heute noch gültig ist. Der vorliegende Beitrag geht in diesem Kontext drei zentralen Problemen nach, die mit dem eingangs beschriebenen Empiriedefizit zusammenhängen: Worin manifestiert sich der festgestellte „Stiefkind-Status“ der Rechnungshöfe in den Sozialwissenschaften? Zeigen sich gegenüber den von Diederich u.a. erkannten Mangelerscheinungen und Kritikpunkten heute wesentliche Veränderungen, oder liegt die sozialwissenschaftlich geprägte Rechnungshofforschung noch immer in einem tiefen „Dornröschenschlaf“? Welche Ursachen sind für die festgestellten Defizite auszumachen, und welche Perspektiven bieten sich für die zukünftige Forschung?

Dazu werden im Folgenden die einzelnen Feststellungen anhand der Forschungsbeiträge zur Unabhängigkeit von Rechnungshöfen überprüft. Diese spielt für viele weiterführende Forschungsfragen rund um die Rechnungshöfe eine wichtige Rolle. Daher eignet sich dieses Themengebiet besonders gut dafür, die Befunde von Diederich u.a. exemplarisch zu untersuchen.

\section{Unabhängigkeit von Rechnungshöfen}

Der Ausgangspunkt der Debatten um die Unabhängigkeit von Rechnungshöfen sind die geltenden gesetzlichen Bestimmungen. Die Landesrechnungshöfe sind entsprechend der Regelungen in den Landesverfassungen und nach $\$ 1$ der Landesrechnungshofgesetze (LRHG) als oberste Landesbehörden „unabhängig“ beziehungsweise „selbstständig“ und nur dem Gesetz unterworfen. Sie unterliegen keinen Weisungen. ${ }^{7}$ Diese gesetzliche Definition ist allerdings nicht geeignet, die Unabhängigkeit von Rechnungshöfen empirisch umfassend zu beschreiben, weshalb in der Literatur viele Ausdifferenzierungen vorgenommen werden.

Im juristischen Schrifttum werden hierzu vor allem zwei Aspekte hervorgehoben. So wird zwischen materieller und personeller Unabhängigkeit unterschieden. ${ }^{8}$ Die materielle Unabhängigkeit bezieht sich auf eine angemessene Ressourcenausstattung der Rechnungshöfe, die nicht zu einer faktischen Begrenzung der Prüfungstätigkeit führen darf. ${ }^{9}$ Die personelle Unabhängigkeit bedeutet, dass die Mitglieder des Rechnungshofes keinen internen oder externen Weisungen - etwa durch den Rechnungshofpräsidenten oder durch die Legislative und Exekutive - unterworfen sind. Dementsprechend ergibt sich die formale Unabhängigkeit der Rechnungshöfe nicht zuletzt aus dem Status der Mitglieder. ${ }^{10}$ Diese

7 Vgl. Hedda von Wedel, Public Financial Control in Europe: The Example of the Federal Republic of Germany, in: Milagros Garcia Crespo (Hrsg.), Public Expenditure Control in Europe, Coordinating Audit Functions in the European Union, Cheltenham u.a. 2005, S. 79 - 98.

8 Vgl. Hans Schäfer, The Constitutional Position of Supreme Audit Institutions with Special Reference to the Federal Republic of Germany, in: Benjamin Geist (Hrsg.), State Audit: Developments in Public Accountability, London / Basingstoke 1993, S. 46 - 69, S. 49.

9 Vgl. Erich Sandl, Reflections on the Perspectives of Financial Control, German Foundation for International Development (DSE), Public Administration Centre (ZÖV) 1987, S. 26.

10 Vgl. Markus Glatfeld, Die Auswirkungen effizienzsteigernder Maßnahmen in der öffentlichen Verwaltung unter besonderer Berücksichtigung von Ausgliederungen beziehungsweise Privatisierungen auf die Arbeit der Rechnungshöfe (Dissertation Technische Hochschule Darmstadt), Darmstadt 1997, S. 21; Rechnungshof Schleswig-Holstein, Grundlagen der Finanzkontrolle und der öffentlichen Haushaltswirtschaft in Schleswig Holstein, Kiel 2003, S. 7. 
Unabhängigkeit soll die Prüfungstätigkeit und die Prüfungsergebnisse in erster Linie vor äußeren Einflüssen schützen, so dass die Finanzkontrollbehörden frei davon sind, „nach Beifall zu schielen" 11 .

Diese eher formale Differenzierung vernachlässigt aber beispielsweise das zum Teil sehr brisante Thema der Besetzungspolitik von Rechnungshöfen, das vor allem empirisch gut greifbar ist. So hat es auch in jüngerer Vergangenheit immer wieder sehr hitzige parlamentarische Debatten um die Bestellung von Rechnungshofpräsidenten oder Vizepräsidenten gegeben. Beispielsweise sind für Landesrechnungshöfe seit 1991 einige Fälle bekannt, wo die vorgeschlagenen Kandidaten in der parlamentarischen Abstimmung nicht die geforderte Mehrheit auf sich vereinigen konnten (etwa Sachsen, Thüringen und Berlin). ${ }^{12}$ Darüber hinaus gab es auch in anderen Bundesländern (etwa Bayern, Brandenburg oder Hessen) Auseinandersetzungen um die Besetzung der Rechnungshofspitze. Hier wurde vor allem die Nähe der Kandidaten zur Exekutive bemängelt. ${ }^{13}$ Diese und ähnliche Sachverhalte lassen sich aber nicht über die formalrechtlichen Bestimmungen dokumentieren und verdeutlichen exemplarisch, wie wichtig empirische Forschung in diesem Bereich ist.

Neben der „materiellen“ und „personellen“ Differenzierung gibt es noch die Unterscheidung zwischen organisatorischer und operationeller Unabhängigkeit ${ }^{14}$, die für die deutsche Rechnungshofliteratur aber eher untypisch ist. Die organisatorische Unabhängigkeit bezieht sich vor allem auf das institutionelle Gefüge, während die operationelle Unabhängigkeit vor allem auf die Prüfungstätigkeit der Rechnungshöfe abhebt. Sie hinterfragt, wie die Unabhängigkeit unter verschiedensten Prüfungsmethoden und -kontakten dauerhaft gesichert werden kann, ohne zu großen Informationsverlusten oder einer zu starken Vereinnahmung durch andere Akteure zu führen.

\subsection{Geringer kumulativer Erkenntniszuwachs}

Viele der bereits bestehenden Aufsätze über die Rechnungshöfe generieren bei genauerem Hinsehen kaum neue Erkenntnisse, beziehungsweise ist der Erkenntniszuwachs aus diesen Untersuchungen vergleichsweise gering. Dies liegt unter anderem daran, dass die meisten Ansätze oftmals nur wiederholt werden, ohne sie kritisch zu hinterfragen und Neuerungen oder Erweiterungen zu entwickeln. Dadurch wird ein langfristiger und signifikanter „kumulativer Erkenntnisgewinn" 15 deutlich erschwert, nicht zuletzt weil insbesondere die

11 Herbert Sauer / Hans Blasius, Politik und Finanzkontrolle durch Rechnungshöfe, in: DVBl, 100. Jg. (1985), H. 10, S. 548 - 556.

12 Vgl. Sächsischer Landtag, Plenarprotokoll 2/15, S. 1028, S. 1037; Thüringer Landtag, Plenarprotokoll 4/40, S. 4042; Abgeordnetenhaus Berlin, Plenarprotokoll 16/54, S. 5087.

13 Vgl. Dieter C. Umbach / Franz-Wilhelm Dollinger, Zwischen Bestenauslese und Demokratieprinzip. Die Besetzung der Landesrechnungshöfe unter Berücksichtigung der Bewerbung, Wahl und Ernennung der Mitglieder des Rechnungshofs Brandenburg, Berlin 2007; Günter Mann, Unabhängige Kontrolleure? Probleme der Besetzung von Kontrollämtern, dargestellt am Beispiel des Leitungspersonals von Rechnungshöfen, in: ZParl, 12. Jg. (1981), H. 3, S. 353 - 367, S. 357.

14 Vgl. Michael Power, The Audit Society. Rituals of Verification, Oxford 1997, S. 132.

15 Werner Jann, Strategien und Probleme der vergleichenden Verwaltungswissenschaft, in: Rainer Koch (Hrsg.), Verwaltungsforschung in Perspektive. Ein Colloqium zur Methode, zum Konzept und zum Transfer, Baden-Baden 1987, S. 39 - 55. 
deutsche Rechnungshofdebatte zum Teil sehr selbstbezogen ist. Allerdings richtet sich diese Kritik keinesfalls an alle zurückliegenden Untersuchungen, da es auch hier Arbeiten gibt, die sich aus gutem Grund zu einem wahren Standard der Rechnungshofliteratur entwickelt haben und die aus dem allgemeinen Forschungsdiskurs kaum noch wegzudenken sind. ${ }^{16}$

Ferner gibt es Beiträge, die eindeutige sozialwissenschaftliche Untersuchungselemente beinhalten und die mit differenzierten abwägenden Betrachtungen durchaus zwischen normativem Anspruch und empirischer Wirklichkeit zu unterscheiden wissen. ${ }^{17}$ Allerdings handelt es sich hierbei nicht direkt um sozialwissenschaftliche Arbeiten, sondern oftmals um juristische Abhandlungen, die mit ihren Fragestellungen den Schwerpunkt bei der zu beobachtenden Wirklichkeit setzen. Auffällig ist überdies, dass auf dem Gebiet der Rechtswissenschaft vor allem jene Untersuchungen neue Wege der Erkenntnis aufzeigen, die sich um alternative oder interdisziplinäre Perspektiven bemühen.

Der tiefere Blick in die bestehende Literatur lässt erkennen, dass vor allem in den sehr stark rechtswissenschaftlich geprägten Untersuchungen der Erkenntnisgehalt in den letzten Jahren merklich nachgelassen hat. Die zentralen Auseinandersetzungen im Zusammenhang mit dem Thema Unabhängigkeit - wie etwa Rechnungshof und Politik ${ }^{18}$, Unabhängigkeit und Wirksamkeit ${ }^{19}$, Stellung der Rechnungshöfe ${ }^{20}$, Rechnungshöfe als Bera-

16 Dazu gehören unter anderem Hans Herbert von Arnim, Grundprobleme der Finanzkontrolle, in: DVBl, 98. Jg. (1983), H. 17, S. 664 - 675; Karl Wittrock, Die Zusammenarbeit der Rechnungshöfe in der Bundesrepublik, Mittel und Zweck überregionaler Kooperation, in: Die Verwaltung, 19. Jg. (1986), H. 3, S. 363 - 372; Hans Blasius, Einige Bemerkungen zu aktuellen Problemen der Finanzkontrolle, in: NWVBl, 14. Jg. (2000), H. 11, S. 413 - 417; Ernst Heuer, Kommentar zum Haushaltsrecht des Bundes und der Länder sowie der Vorschriften zur Finanzkontrolle, Neuwied 2007.

17 Vgl. Ulrich Battis, Rechnungshof und Politik, in: DÖV, 29. Jg. (1976), H. 21, S. 721 - 727, S. 724 ff.; Karl Wittrock, Parlament, Regierung und Rechnungshof. Zur Geschichte einer schwierigen Dreiecksbeziehung, in: ZParl, 17. Jg. (1986), H. 3, S. 414 - 422; Hans Herbert von Arnim, a.a.O. (Fn. 16).

18 Vgl. Gunter Kisker, Rechnungshof und Politik, in: Hans Herbert von Arnim (Hrsg.), Finanzkontrolle im Wandel. Vorträge und Diskussionsbeiträge der 15. Verwaltungswissenschaftlichen Arbeitstagung 1988 des Forschungsinstituts für öffentliche Verwaltung bei der Hochschule für Verwaltungswissenschaften Speyer, Berlin 1989, S. 195 - 219; Susanne Tiemann, Nochmals: Rechnungshof und Politik. Entgegnung auf Battis, in: DÖV, 30. Jg. (1977), H. 7, S. 240 - 243; Ulrich Battis, a.a.O. (Fn. 17).

19 Vgl. Hans-Ernst Folz / Regina Herrmann, Die richterliche Unabhängigkeit der Mitglieder der Rechnungshöfe, in: JZ, 28. Jg. (1973), H. 23/24, S. 769 - 771; Hans Blasius, Zur Unabhängigkeit der Mitglieder der Rechnungshöfe, in: VR, 36. Jg. (1990), H. 4, S. 124 - 129; Udo Müller, Die institutionelle Unabhängigkeit der Rechnungshöfe - Verfassungsmäßige Bedeutung und Gefährdungen im Rahmen der Bemühungen um haushaltswirtschaftliche Konsolidierungen, in: DVBl, 109. Jg. (1994), H. 22, S. $1276-1283$.

20 Vgl. Hans Blasius, Die Bedeutung der Finanzkontrolle und die Stellung der Rechnungshöfe, Teil 1 und Teil 2, in: VOP, 13. Jg. (1991), H. 2 beziehungsweise H. 3, S. 87 - 90 beziehungsweise S. 158 - 163; Hartwig Schlegelberger, Aufgabe und Stellung des Rechnungshofes in unserem Finanzkontrollsystem heute und morgen, in: ders. / Wolfgang Böning / Albert von Mutius (Hrsg.), Finanzkontrolle im föderativen Staat, Heidelberg / Hamburg 1982, S. 4 - 23; Harald Schulze, Stellung und Funktion der Rechnungshöfe im Wandel?, in: Gunther Engelhardt / ders. / Werner Thieme (Hrsg.), Stellung und Funktion der Rechnungshöfe im Wandel, Baden-Baden 1993, S. $33-42$. 
$\operatorname{ter}^{21}$, Wirtschaftlichkeit und Sparsamkeit ${ }^{22}$ - überschneiden sich vielfach und scheinen sich in regelmäßigen Abständen zu wiederholen. Dementsprechend könnten die Berücksichtigung von neuen Perspektiven und die Durchführung von empirischen Analysen und damit die Identifikation von Ursache-Wirkungsbeziehungen einen wesentlichen Beitrag für den Erkenntnisfortschritt leisten. Hierzu gehören etwa systematische empirische Untersuchungen zur Besetzung der Rechnungshofspitzen, Erhebungen über die Prüfungsund Beratungstätigkeit von Rechnungshöfen, die Interaktion von Parlament und Rechnungshof sowie mit dem Adressaten der Prüfberichte. Dabei könnten insbesondere ländervergleichende Studien zusätzliche Erkenntnisse liefern und der Entwicklung neuer Hypothesen dienen. ${ }^{23}$

\subsection{Nur wenige empirische Beweisführungen}

Der von Diederich u.a. beschriebene Mangel an empirischen Beweisen lässt sich auch heute für die Diskussions- und Forschungsbeiträge zur Unabhängigkeit von Rechnungshöfen feststellen. Dabei wird dieser Weg der Erkenntnisgewinnung in vielen Untersuchungen grundsätzlich nicht angestrebt. Hinzu kommt außerdem, dass sich über die Jahre ein Empiriedefizit entwickelt hat, das bestehende Tatsachen mehr oder weniger verschleiert und anerkannte Forschungsmethoden bestenfalls rudimentär verwendet. Dies impliziert neben dem Mangel an innovativen Forschungskonzepten und neueren Forschungsergebnissen vor allem eine begrenzte selbstkritische Auseinandersetzung mit den eigenen Erkenntnissen.

Im Vordergrund steht hier exemplarisch der allzu leichtfertige und unkritische Umgang mit unbewiesenen Hypothesen oder Annahmen. Dies lässt sich zum einen dadurch erklären, dass viele der kursierenden Vermutungen eher implizit formuliert werden. Zum anderen aber werden bestimmte als Erkenntnisse wahrgenommene Aussagen ohne kritische Reflexion einfach als allgemeingültiger Wissensbestand übernommen. Eine eingehende Überprüfung bleibt zumeist aus. Dies führt letztlich zu „Aussagen [...], die sich die meisten empirisch orientierten Sozialwissenschaftler nicht zutrauen würden"24.

Ein prägnantes Beispiel für den Umgang mit Hypothesen sind etwa die zahlreichen immer wieder postulierten Kausalzusammenhänge zwischen der Unabhängigkeit und anderen Eigenschaften von Rechnungshöfen. Dabei wird der Unabhängigkeit a priori stets eine positive Wirkung zugeschrieben, wobei sich nur die wenigsten Aufsätze differenziert mit diesem Forschungsproblem befassen. Beispielsweise konstatiert Karl Wittrock für den Bundes-

21 Vgl. Holger Miß, Die Unabhängigkeit von Bundesrechnungshof und Abschlussprüfung vor dem Hintergrund paralleler Prüfung und Beratung. Eine vergleichende Untersuchung, Baden-Baden 2006, S. 45 - 64; Peter Jakobs-Woltering, Qualifikation der Mitglieder den Veränderungen anpassen. Personal der Rechnungshöfe übernimmt Beratungsfunktion, in: VOP, 18. Jg. (1996), H. 3/4, S. $31-35$.

22 Vgl. Herbert Rischer, Finanzkontrolle staatlichen Handelns. Wirtschaftlichkeit und Sparsamkeit als Prüfungsmaßstäbe, Heidelberg 1995.

23 Vgl. Dirk Berg-Schlosser / Ferdinand Müller-Rommel, Perspektiven der vergleichenden Politikwissenschaft, in: dies. (Hrsg.), Vergleichende Politikwissenschaft, Opladen 2003, S. 331 - 342; Franz Lehner / Ulrich Widmaier, Vergleichende Regierungslehre, Opladen 1995, S. 57.

24 Jörg Bogumil / Werner Jann, Verwaltung und Verwaltungswissenschaft in Deutschland. Einführung in die Verwaltungswissenschaft, Wiesbaden 2009, S. 33. 
rechnungshof eine gewisse, nicht näher definierte Beziehung zwischen Unabhängigkeit und Wirksamkeit: „Die Unabhängigkeit des Bundesrechnungshofes und seiner Mitglieder ist kein Privileg und keine persönliche Auszeichnung der Mitglieder des Bundesrechnungshofes. Sie ist ihnen verliehen, weil dies der Wirksamkeit der Finanzkontrolle dient. "25 Obgleich sich hieraus nicht zwingend eine Hypothese herauslesen lässt, so ist doch die Vermutung eines Zusammenhangs zwischen Unabhängigkeit und Wirksamkeit sehr naheliegend.

Noch deutlichere Aussagen werden für die Zusammenhänge zwischen Unabhängigkeit, Qualität und Effizienz von Kontrollen getroffen. Demnach wird „die Korrelation zwischen Unabhängigkeit des RH [Abkürzung im Original] und Effizienz der Kontrolle auch von finanzwissenschaftlicher und parlamentarischer Seite (an)erkannt "26. Allerdings gibt es bisher keinerlei empirische Erkenntnisse oder Beweise, die diesen Zusammenhang untermauern oder bestätigen. Und so ist in Bezug auf Unabhängigkeit und Wirksamkeit von Rechnungshöfen weder bekannt, ob es sich um einen positiven oder einen negativen sowie um einen starken oder schwachen Zusammenhang handelt. Beides müsste sich jedoch theoretisch feststellen oder ableiten lassen, wenn es sich um grundlegend empirische Untersuchungen handeln würde. Insofern bleibt diese Behauptung einer „Korrelation“ zwischen Unabhängigkeit und Kontrolleffizienz den Beweis schuldig. Dies gilt ungeachtet der Tatsache, dass es äußerst schwierig sein dürfte, die Effizienz von Rechnungshofkontrollen zu ermitteln. Derzeit gibt es hierzu weder Konzepte noch Ideen, wie derartige Forschungsvorhaben adäquat umgesetzt werden könnten.

Die Diskussion um pseudo-empirische Ergebnisse erschöpft sich aber nicht in den bisher genannten Zusammenhängen. Ein anderes Beispiel postuliert sogar einen direkten $\mathrm{Zu}$ sammenhang zwischen „dem Grad der dem Kontrolleur eingeräumten Unabhängigkeit und der Qualität der Kontrollergebnisse"27. Diese Behauptung wirft gleich mehrere Fragen auf. Beispielsweise bleibt unklar, was die „Qualität der Kontrollergebnisse“ überhaupt ist, zumal Qualität kein unidimensionales Konstrukt ist. ${ }^{28}$ Ferner dürfte es recht schwer fallen, ein qualitativ hochwertiges von einem qualitativ minderwertigen Prüfungsergebnis zu unterscheiden, denn Qualitäten von Rechnungshofprüfungen sind empirisch nicht ohne Weiteres zu operationalisieren. Hinzu kommt, dass auch immer nur ein bestimmter Anteil der Prüfungsergebnisse veröffentlicht wird ${ }^{29}$, was Probleme der Fallauswahl nach sich zieht. Es

25 Karl Wittrock, Warum muss Finanzkontrolle unabhängig sein?, in: DVBl, 99. Jg. (1984), H. 16, S. $823-826$.

26 Hans Blasius / Horst Kühne, Stärken und Schwächen des Rechnungshofs, in: DÖV, 44. Jg. (1991), H. 10, S. $393-401$.

27 Holger Miß, a.a.O. (Fn. 21), S. 45.

28 Vgl. dazu exemplarisch die Qualitätsdiskussionen über Hochschulen und Fernsehangebote: Peer Pasternack, Kennziffern und Indikatoren. Politische und Soziale Sprengkraft, in: Karin Zimmermann / Sigrid Metz-Göckel / Marion Kamphans (Hrsg.), Perspektiven der Hochschulforschung, Wiesbaden 2008, S. 353 - 362; Miriam Tebert, Erfolg durch Qualität. Programmcontrolling beim WDR Fernsehen, in: Media Perspektiven 2000, H. 2, S. 85 - 93.

29 Vgl. Heinz Günter Zavelberg, Staatliche Finanzkontrolle und Wirtschaftsprüfung, in: Wirtschaftsprüferkammer Mitteilungen, 29. Jg. (1992), S. 241 - 245. Der Präsident des Bayerischen Rechnungshofes spricht beispielsweise davon, dass in seinem Haus rund 90 Prozent der Prüfungsergebnisse nicht veröffentlich werden. Heinz Fischer-Heidlberger, Vortrag des Präsidenten des Bayerischen Obersten Rechnungshofs zum Thema: „Der ORH - Interessenvertreter für den Steuerzahler" am 28. Februar 2007, http://www.orh.bayern.de/files/Reden/Rede Gemeinde Immanuel A4.pdf (Abruf am 10. April 2008). 
gibt zweifelsohne noch weitere nennens- und lesenswerte Beispiele ${ }^{30}$, auf deren Darstellung an dieser Stelle aber verzichtet wird.

Zusammenfassend lässt sich feststellen, dass trotz der jüngeren und vereinzelt auch empirisch ausgerichteten Literatur begründete Annahmen oder Vermutungen sowie die kritische Auseinandersetzung mit der gängigen Literatur Seltenheitswert haben. Daher sollte die Forschung über Rechnungshöfe viel häufiger auf sozialwissenschaftliche Erhebungs- und Auswertungsmethoden zurückgreifen und die zur Verfügung stehenden Datenquellen stärker nutzen, um dieses Empiriedefizit zu schließen. Nicht ohne Grund spricht Heinz Günter Zavelberg im Hinblick auf die Prüfungsberichte und die daraufhin getroffenen parlamentarischen Entscheidungen von einer „bedeutsamen Materialsammlung“31.

\subsection{Unzulängliche und selektive Beweisführungen}

Das Problem der Verzerrung von Ergebnissen durch die getroffene Fallauswahl („selection

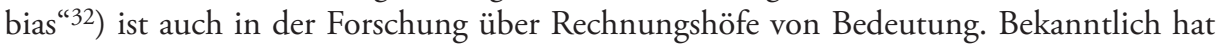
eine theoretische Fundierung der Fallauswahl ganz andere Implikationen als eine willkürliche Auswahl von Fällen. Dabei ist generell unstrittig, dass Sondereinflüsse oder Abhängigkeiten durch einzelne Fälle auch in theoretisch begründeten Vorgehensweisen nie ganz auszuschließen sind. ${ }^{33}$

In der wissenschaftlichen Auseinandersetzung über Rechnungshöfe gibt es hin und wieder aber Anhaltspunkte, die auf eine fragwürdige Fallauswahl ohne theoretisch-analytische Grundlage hindeuten. So vergleicht Peter Haller beispielsweise die Rechnungshöfe von Ghana und Simbabwe mit Deutschland, Seung-Cheol Yi die der Republik Korea und Deutschlands. ${ }^{34}$ In beiden Untersuchungen fehlt eine plausible Begründung, warum gerade diese Institutionen für die vergleichende Analyse von besonderer Bedeutung sind. Es handelt sich folglich eher um Vergleiche im wörtlichen Sinn, das heißt das Erkennen von Unterschieden und Gemeinsamkeiten. Der Vergleich im analytischen Sinn ist aber neben dem

30 Vgl. Heinz Günter Zavelberg, Prüfungsaufträge und Unabhängigkeit der Rechnungshöfe, in: DÖV, 32. Jg. (1979), H. 19, S. 705 - 708; Hans Blasius, a.a.O. (Fn. 19), S. 124.

31 Vgl. Heinz Günter Zavelberg, Staatliche Finanzkontrolle in Deutschland. Über Arbeit und Effektivität des Bundesrechnungshofes, in: Die Verwaltung, 28. Jg. (1995), H. 4, S. $534-535$.

32 So listet beispielsweise Uwe Wagschal fünf mögliche Quellen für einen „selection bias“ auf: (1) die Auswahl der untersuchten Länder, (2) die Auswahl und Operationalisierung der erklärenden (unabhängigen) Variablen, (3) die Auswahl und Operationalisierung der zu erklärenden (abhängigen) Variablen, (4) die Wahl der Untersuchungsperiode und schließlich (5) die Art des angewandten Vergleichs. Vgl. Uwe Wagschal, Staatsverschuldung, Ursachen im internationalen Vergleich, Opladen, 1996, S. 16.

33 Vgl. Hans-Joachim Lauth / Jürgen R. Winkler, Methoden der Vergleichenden Politikwissenschaft, in: Hans-Joachim Lauth (Hrsg.), Vergleichende Regierungslehre. Eine Einführung, Wiesbaden 2002, S. 37 - 69, S. 56; Dirk Berg-Schlosser / Ferdinand Müller-Rommel, a.a.O. (Fn. 23), S. 334; W. Phillips Shively, Case Selection. Insights from Rethinking Social Inquiry, in: Political Analysis, 14. Jg. (2006), H. 3, S. $344-347$, S. 345.

34 Vgl. Peter Haller, Rechnungshöfe in Industrie- und Entwicklungsländern. Ein Vergleich, Frankfurt am Main u.a. 2000; Seung-Cheol Yi, Moderne Finanzkontrolle. Eine vergleichende Bewertung der obersten Finanzkontrollbehörden in der Bundesrepublik Deutschland und der Republik Korea, Pfaffenweiler 1987. 
Pflichtprogramm der Ermittlung von Unterschieden und Gemeinsamkeiten vor allem an den Wirkungen von bestimmten Merkmalen/Variablen (bei x-zentrierter Forschung) oder an der Erklärung von Ergebnissen sowie Ereignissen (bei y-zentrierter Forschung) interessiert. $^{35}$

Neben diesen Problemen gibt es eine Reihe anderer Schwierigkeiten, die symptomatisch sind für die mangelhafte Durchführung von empirischen Untersuchungen über Rechnungshöfe. So steht etwa dem intensiven Umgang mit dem rechtswissenschaftlichen Gedankengut ein nahezu fahrlässiger Umgang mit empirischen Daten, Auswertungen und Schlussfolgerungen gegenüber. Bisher gibt es in der gängigen Literatur über Rechnungshöfe keine Auseinandersetzung mit den Grundlagen und Problemen des Erklärens sozialer oder institutioneller Phänomene oder damit, wie Forschungsfragen adäquat beantwortet werden können. ${ }^{36}$ Für die empirische Rechnungshofforschung sollte es daher insbesondere darum gehen, bestehende Hypothesen gegebenenfalls zu falsifizieren und lieb gewonnene Vorurteile zu widerlegen. Wissenschaftliche Erklärungen liegen insbesondere dann vor, wenn bestimmte Standards erfüllt werden: etwa eine eindeutige Struktur der Argumentation, logische Korrektheit oder die empirische Begründbarkeit. ${ }^{37}$

Darüber hinaus gibt es über Rechnungshöfe kaum Datenerhebungen oder -sammlungen. Die wenigen vorhandenen empirischen Untersuchungen sind mitunter unzureichend und punktuell. Es sei jedoch darauf hingewiesen, dass dies nicht bei allen Arbeiten der Fall ist. Insbesondere die Rechnungshöfe selbst haben in diversen Publikationen hin und wieder Daten vorgelegt, die bestimmte Entwicklungen auch über längere Zeiträume gut dokumentieren. ${ }^{38}$ Allerdings scheitern diese Darstellungen zumeist an weiterführenden Anschlussuntersuchungen oder an vergleichenden Betrachtungen. Viele der Studien zielen letztlich auch nicht direkt darauf ab, den Forschungsgegenstand Rechnungshof in einen größeren Kontext zu stellen. Die meisten dienen ausschließlich deskriptiven Zwecken. Folglich empfiehlt sich neben den oben genannten Erhebungsmethoden auch ein Rückgriff auf sozialwissenschaftliche Forschungskonzepte, die eine klare und operationalisierbare Sprache zugrunde legen. Dazu gehört die Umsetzung einer theorie- und hypothesengeleiteten Vorgehensweise, die bei einer plausiblen Forschungsfrage beginnt und bei substanziellen Erkenntnissen über mögliche Kausalbeziehungen der Finanzkontrolle endet.

35 Vgl. Steffen Ganghof, Kausale Perspektiven in der vergleichenden Politikwissenschaft: X-zentrierte und Y-zentrierte Forschungsdesigns, in: Sabine Kropp / Michael Minkenberg (Hrsg.), Vergleichen in der Politikwissenschaft, Wiesbaden 2005, S. 76 - 93.

36 Vgl. Thomas Gschwend / Frank Schimmelpfennig, Lehren für den Dialog zwischen Theorie und Daten, in: dies. (Hrsg.), Forschungsdesign in der Politikwissenschaft. Probleme - Strategien Anwendungen, Frankfurt am Main / New York 2007, S. 327 - 340; Franz Lehner / Ulrich Widmaier, a.a.O. (Fn. 23), S. 54.

37 Vgl. Rainer Schnell / Paul Hill / Elke Esser, Methoden der empirischen Sozialforschung, Oldenbourg / München 2005, S. 57.

38 Vgl. Hans Hermann Bücker, Personalwirtschaft beim Landesrechnungshof Nordrhein-Westfalen, in: Rechnungshof Nordrhein-Westfalen (Hrsg.), 40 Jahre Landesrechnungshof Nordrhein-Westfalen 1948-1988, Düsseldorf 1988, S. 51 - 70; Otto-Günther Lonhard, Der Rechnungshof Baden-Württemberg, in: Rechnungshof Baden-Württemberg (Hrsg.), Finanzkontrolle in BadenWürttemberg - Beiträge zu Geschichte, Aufgaben und Arbeit der Rechnungsprüfungsbehörden, Karlsruhe 1987, S. $111-143$. 


\subsection{Stark normativ geprägter Forschungsdiskurs}

Die extreme Präsenz juristischer Ansätze und Denkmuster führte in der Vergangenheit zu normativ aufgeladenen Auseinandersetzungen, bei denen es oft um das Für und Wider zweier oder mehrerer konkurrierender Rechtsauffassungen ging. Diese Untersuchungen befassen sich vornehmlich mit so genannten „Scholastikerproblemen “39, die sich vor allem an den bestehenden Regelwerken und Vorschriften orientieren.

Es existieren bisher nur vereinzelte Ansätze, die diese normativen Diskussionen aufbrechen und konkrete empirische Erkenntnisse erarbeiten. ${ }^{40}$ Zwar gibt es zahlreiche Beiträge, die eine Synthese zwischen normativen Annahmen und Empirie versuchen, doch leiden diese Untersuchungen zumeist an einer grundlegenden Theorie- und Methodenarmut, gehen oftmals nicht über anekdotische Evidenz hinaus. Daher verwundert es kaum, dass sich die zentralen Auseinandersetzungen entlang normativer, aber entgegengesetzter Argumentationsstränge bewegen. Dies zeigt sich an verschiedenen Diskussionen über die Tätigkeit der Rechnungshöfe und den potenziellen Auswirkungen auf deren Unabhängigkeit.

Einige Autoren befürworten beispielsweise eine Beratung durch Rechnungshöfe und sehen darin eine zulässige Hilfestellung. Andere wiederum lehnen dieses Vorgehen als verfassungsrechtlich bedenklich ab. ${ }^{41}$ Auch für das politische Wirken gibt es vergleichbare Diskussionsstränge. Während einige Wissenschaftler und Praktiker es als geradezu zwangsläufig sehen, dass bestimmte Erkenntnisse der Rechnungshöfe auch politische Wirkungen entfalten, sehen die Kritiker hier eine unzulässige Einmischung und fordern absolute politische Enthaltsamkeit für die Rechnungshöfe. ${ }^{42}$ Ähnlich ist es mit Prüfungsersuchen durch die Parlamente. Während die eine Seite sich eine breitere Informationsbasis erhofft, befürchten die Kritiker vor allem eine Instrumentalisierung durch die Opposition oder ein „Lahmlegen" des Rechnungshofes durch die regierungstragende Mehrheit.

39 Vgl. Gottfried-Ernst Mahrenholz, Festrede des Vizepräsidenten des Bundesverfassungsgerichtes (i. R.), in: Landtag Niedersachsen (Hrsg.), Festakt zum 50-jährigen Bestehen des Niedersächsischen Landesrechnungshofes, Hannover 1998, S. 15.

40 Vgl. beispielsweise Peter Jakobs-Woltering, Die Personalqualifikation und Personalentwicklung der Rechnungshöfe: Ein Problem der Erfolgskontrolle. Ein theoretisch-empirischer Beitrag zur Personalforschung, Frankfurt am Main u.a. 1993; Christopher Pollitt / Hilkka Summa, Reflexive Watchdogs? How Supreme Audit Institutions Account For Themselves, in: Public Administration, 75. Jg. (1997), H. 2, S. 313 - 336; Stefan Bajohr, Parlamentarische Prüfung von Wirtschaftlichkeit und Sparsamkeit der Rechnungshöfe, in: VR, 46. Jg. (2000), H. 12, S. 406 - 410; Markus Seyfried, Die Unabhängigkeit und Wirksamkeit von Landesrechnungshöfen - Eine empirisch vergleichende Bestandsaufnahme, Wiesbaden 2011.

41 Vgl. Michael Freytag, Der Europäische Rechnungshof, Institution, Funktion und politische Wirkung. Integration Europas und Ordnung der Weltwirtschaft, Baden-Baden 2005, S. 47; Hans Blasius, Finanzkontrolle und Gesetzgebung - Können die Rechnungshöfe einen Beitrag zur Gesetzgebung leisten?, in: DÖV, 42. Jg. (1989), H. 7, S. 298 - 306, S. 304.

42 Vgl. Eberhard Fricke, Bedarf es eines Anstoßes zu einer erneuten wissenschaftlichen Stabilisierung der staatlichen Finanzkontrolle?, in: DÖV, 61. Jg. (2008), H. 6, S. 226 - 234, S. 234; Wolfgang Kitterer, Kann die Finanzkontrolle dazu beitragen, die Funktionsfähigkeit der Demokratie zu stärken? Gedanken aus finanzwissenschaftlicher Sicht, in: Wolfgang Böning / Albert von Mutius (Hrsg.), Finanzkontrolle im repräsentativ-demokratischen System, Heidelberg 1989, S. 173 179, S. 175 f. 
Dabei ist insbesondere die Rolle der Rechnungshöfe als Dialogpartner der Parlamente ein Paradebeispiel für mehr empirischen Forschungsbedarf und für ein Loslösen von normativen Gedankengerüsten. Auf den ersten Blick ist dieses Verhältnis geprägt durch den Gegensatz von regierungstragender Mehrheit und Opposition. Dementsprechend kann es innerhalb des Parlaments durchaus Bestrebungen geben, eine zu kritische Kontrolle der Exekutive zu unterbinden, was letztlich durch die Mehrheitsverhältnisse im Haushaltsausschuss, der etwa die Beschlussempfehlungen zu den Prüfberichten der Rechnungshöfe verfasst, ohne weiteres denkbar wäre. Dass von dieser Möglichkeit jedoch nur wenig Gebrauch gemacht wird, lässt sich direkt aus den Beschlussempfehlungen der Haushaltsausschüsse zum Jahresbericht der Rechnungshöfe herauslesen. ${ }^{43}$ Darüber hinaus ist das Parlament auf die Zusammenarbeit mit den Rechnungshöfen angewiesen, wenn es seiner Kontrollfunktion gegenüber der Exekutive gerecht werden will. Insofern hat es und insbesondere die regierungstragende Mehrheit kein generelles Interesse daran, sich durch eine dauerhafte Blockadehaltung einer der zentralen Grundlagen parlamentarischer Finanzkontrolle zu berauben. ${ }^{44}$ Im Gegenteil, es existiert ein gewisses Vertrauensverhältnis zwischen Parlament und Rechnungshof ${ }^{45}$, das sich aber kaum durch normative Rechtsinterpretation erfassen lässt. Auch hier liegen die Möglichkeiten vor allem in Erhebungsinstrumenten wie etwa Interviews oder Befragungen. Dieses Beispiel illustriert nochmals die zentrale Bedeutung, die der empirischen Forschung in diesem Feld zukommen kann, denn ähnliche Lücken zwischen normativen Annahmen und Empirie lassen sich wie gezeigt auch für andere Forschungsfragen über Rechnungshöfe dokumentieren.

\section{Erklärungsversuche und Perspektiven für das Dasein als Stiefkind}

Die Feststellungen von Diederich u.a. haben gegenwärtig nichts von ihrer Gültigkeit verloren. Dabei stellt sich vor allem die Frage, warum die Rechnungshöfe bis heute nur sehr selektiv in das Bewusstsein der Wissenschaft gerückt sind. Zwei zentrale Gründe sind zu nennen: erstens die eingeschränkte Nutzung des Feld- und Informationszugangs und zweitens die selbst auferlegte politische Zurückhaltung der Rechnungshöfe.

Der Feld- und Informationszugang ist zwar noch verbesserungsbedürftig, doch gibt es bereits eine Vielzahl von Informationen über Rechnungshöfe, die öffentlich zugänglich und auswertbar sind. Dennoch ist die Datenlage zum gegenwärtigen Zeitpunkt nur mangelhaft aufgearbeitet und reflektiert. Selbst in der Forschungsliteratur wird auf diesen Missstand hingewiesen. ${ }^{46}$ Die Kritik am schlechten Datenbestand bezieht sich sowohl auf die Institution Rechnungshof (das heißt Ressourcen, Ausstattung etc.) als auch auf ihre Tätigkeit (das heißt Prüfung, Beratung etc.). Hinzu kommt, dass die Rechnungshöfe oft vergangenheitsbezogen arbeiten und ein Großteil ihres Wirkens im Verborgenen bleibt beziehungsweise

43 Vgl. Markus Seyfried, a.a.O. (Fn. 40), S. $176 \mathrm{ff}$.

44 Vgl. Stefan Bajohr, Perspektiven der Finanzkontrolle: Parlamentarische Prüfungsaufträge an Rechnungshöfe, in: Verwaltungsarchiv, 91. Jg. (2000), H. 4, S. 507 - 539, S. 515.

45 Vgl. Franz Fiedler, The Independence of Supreme Audit Institutions, in: INTOSAI (Hrsg.), 50 years (1953-2003). A Special Publication of the International Organization of Supreme Audit Institutions, Wien 2004, S. $114 \mathrm{f}$.

46 Vgl. Wolfgang Göke, Der Niedersächsische Landesrechnungshof: Wandel und Struktur der Finanzkontrolle in Niedersachsen, in: NdsVBl, 8. Jg. (2001), H. 3, S. $62-67$. 
die Prüfungstätigkeit und ihre Folgen ohnehin nur sehr schwer messbar sind. ${ }^{47}$ Allerdings sprechen diese bestehenden Einschränkungen abermals für das reichhaltige Methodenrepertoire der Sozialwissenschaften, denn dazu gehört letztlich auch das Erschließen von Quellen, die nicht unmittelbar öffentlich zugänglich sind.

Der zweite eher inhaltliche Grund für das Dasein als Stiefkind liegt in der selbst auferlegten Zurückhaltung der Rechnungshöfe gegenüber politischen Verstrickungen und in der institutionellen Ausgestaltung der Prüfungstätigkeit. Dieses so genannte „political self restraint “ ${ }^{48}$ soll die Neutralität des Rechnungshofes im Sinne eines unpolitischen Akteurs unterstreichen. Im schlimmsten Fall kann diese politische Tabuisierung zu einer Kontrollbeschränkung führen, wodurch interessante sowie umstrittene Inhalte und Ansichten nur schwer zugänglich werden. ${ }^{49}$ Zudem stellt sich die Frage, warum eine Institution, die strittigerweise hin und wieder als ,apolitisch" definiert wird ${ }^{50}$ und der ein konkretes Sanktionspotenzial fehlt ${ }^{51}$, auf großes Forschungsinteresse in einem Bereich stoßen sollte, bei dem es oftmals gerade um Normen und Regeln, Politikprozesse und Politikinhalte geht. Dem lässt sich jedoch entgegenhalten, dass unabhängige Zentralbanken oder Verfassungsgerichte sehr stark im Fokus der Sozialwissenschaften stehen. ${ }^{52}$ Hierbei dürfte aber erneut die Erreichbarkeit von Daten oder Informationen eine wichtige Rolle spielen. Die Wirksamkeit der unabhängigen Zentralbank lässt sich beispielsweise viel einfacher erfassen (etwa anhand der festgelegten Zinssätze oder der Höhe der Inflationsrate). Solche „harten“ Indikatoren gibt es aber für die Messung oder Erfassung der Prüfungsleistung und der Wirkung von Rechnungshöfen nicht oder bestenfalls vereinzelt. ${ }^{53}$ In diesem Kontext ge-

47 Vgl. Rudolf Dieckmann, Zukunftsperspektiven eines Rechnungshofes, in: DÖV, 45. Jg. (1992), H. 21, S. 893 - 903, S. 899; Heinrich Reinermann, Messungsprobleme der Rechnungskontrolle, in: Die Verwaltung, 14. Jg. (1981), H. 4, S. $483-500$.

$48 \mathrm{Vgl}$. Hariolf M. Wenzler, Institutionenökonomik und öffentliche Finanzkontrolle. Eine Analyse am Beispiel der Europäischen Union, Frankfurt am Main 1996, S. 149; Franz-Otto Gilles, Die verkannte Macht. Determinanten der Nachkriegsgeschichte der Institution Rechnungshof, Berlin 1986, S. 234; Hedda Czasche-Meseke, The Influence of the German Federal Court of Audit (FCA) on Policy Decisions, in: Managerial Auditing Journal, 10. Jg. (1995), H. 9, S. 5 - 12.

49 Vgl. Michael Freytag, a.a.O. (Fn. 41), S. 243.

50 Vgl. Henrik Jeep, Szientifische Norm oder politische Legitimation? Das Problem der Wirtschaftlichkeit in der Staatsadministration aus wirtschaftswissenschaftlicher Sicht, in: Theo Pirker (Hrsg.), Autonomie und Kontrolle. Beiträge zur Soziologie des Finanz- und Steuerstaates, Berlin 1989, S. 106 - 133, S. 125; Harald Schulze, Stellung und Funktion der Rechnungshöfe im Wandel?, in: Gunther Engelhardt / ders. / Werner Thieme (Hrsg.), Stellung und Funktion der Rechnungshöfe im Wandel?, Baden-Baden 1993, S. 33 - 42, S. 37.

51 Vgl. Helmut Schulze-Fielitz, Kontrolle der Verwaltung durch Rechnungshöfe, in: VVDStRl 1996, H. 55, Berlin / New York, S. 233 - 277, S. 237; Hariolf M. Wenzler, a.a.O. (Fn. 48), S. 145.

52 Für Zentralbanken: Alex Cukierman / Steven B. Webb, Political Influence on the Central Bank: International Evidence, in: World Bank Economic Review, 9. Jg. (1995), H. 3, S. 397 - 423; dies. I Bilin Neyapti, Measuring the Independence of Central Banks and its Effect on Policy Outcomes, in: World Bank Economic Review, 6. Jg. (1992), H. 3, S. 353 - 398; für Verfassungsgerichte: Georg Vanberg, Legislative-Judicial Relations: A Game-Theoretic Approach to Constitutional Review, in: American Journal of Political Science, 45. Jg. (2001), H. 2, S. 346 - 361; ders., Verfassungsgerichtsbarkeit und Gesetzgebung: Zum politischen Spielraum des Bundesverfassungsgerichts, in: Steffen Ganghof / Philip Manow (Hrsg.), Mechanismen der Politik: Strategische Interaktionen im deutschen Regierungssystem, Frankfurt am Main 2005, S. 183 - 213.

53 Vgl. etwa die finanziellen Auswirkungen der Prüfungstätigkeit des Landesrechnungshofes Nordrhein-Westfalen (siehe Lt-Drs. 11/7733, S. 3 ff.; Lt-Drs. 11/8516, S. 3 ff.; Lt-Drs. 12/984, 
winnen Leistungsdokumentationen (wie etwa die Ergebnisberichte zu den Jahresberich$\operatorname{ten}^{54}$ ) sehr stark an Bedeutung. Außerdem könnten die weiter oben erwähnten alternativen Methoden neue und interessante Forschungsansätze und Erkenntnisse über Rechnungshöfe hervorbringen. Die Veränderung der Informationslage und die Möglichkeit für die Erforschung von Rechnungshöfen in Beziehungsgeflechten mit anderen Akteuren durch komplexe Methoden bieten Gelegenheit, das Empiriedefizit zu beseitigen.

\section{Neuausrichtung der Forschung über Rechnungshöfe}

Nach vielen Jahren der Vernachlässigung durch die Sozialwissenschaften liegt die Vermutung nahe, dass der Rechnungshof als Forschungsgegenstand und Forschungslücke von den Vertretern der Disziplin (wieder)entdeckt wurde. Schließlich gibt es heute nur noch sehr wenige Forschungsfelder, die weithin als „unbesetzt“ gelten. Dennoch fällt das Gesamtbild der vorliegenden Bestandsaufnahme eher ernüchternd aus. Immer noch ist die Literatur durch einen abnehmenden kumulativen Erkenntnisgewinn, durch wenig empirische, mangelhafte sowie selektive Forschung und nicht zuletzt durch stark normativ geprägte Diskurse gekennzeichnet. Zwar gibt es immer wieder erfreuliche Ausnahmen, aber insgesamt zeigen sich kaum nennenswerte Veränderungen zu diesen Befunden.

Hinweise auf die bestehenden Defizite und den Entwicklungsbedarf der Rechnungshofliteratur kommen vor allem aus den Sozialwissenschaften. ${ }^{55}$ Dabei kann gerade deren reichhaltiges Methodenrepertoire zu erheblichen Erkenntnisgewinnen beitragen. Somit soll hier vor allem für deutlich mehr empirische Forschung über Rechnungshöfe plädiert werden. Insbesondere die umfangreichen sozialwissenschaftlichen Methoden und Konzepte sowie die verschiedenen Verfahren zur Datenerhebung und -analyse bieten, wie gezeigt, vielfältige Möglichkeiten, die Forschung über Rechnungshöfe neu auszurichten. Dies impliziert selbstverständlich die den Sozialwissenschaften innewohnenden Diskussionen über Methoden, Fallauswahl, Operationalisierung etc. Die neue Ausrichtung bietet aber auch die Gelegenheit für umfangreiche Erkenntnisgewinne etwa über Innensichten, Beziehungen zwischen Akteuren, Determinanten und Bedingungen des parlamentarischen Kontrollerfolgs und dürfte damit viel stärker dem Wesen und den Ursache-Wirkungs-Mechanismen der Finanzkontrolle auf die Spur kommen.

S. 3 ff.; Lt-Drs. 12/1966, S. 3 ff.; Lt-Drs. 12/3315, S. 3 ff.; Lt-Drs. 12/3915, S. 3 ff.; Lt-Drs. 12/4810, S. 3 ff. oder die zugehörigen Prüfungsberichte) oder die Prüfungsdaten des Rechnungshofes Sachsen-Anhalt (http://www.lrh.sachsen-anhalt.de/index.php?id=49 (Abruf am 16. August 2010).

54 Vgl. exemplarisch: Rechnungshof Hansestadt Hamburg, Ergebnisbericht 2007; Rechnungshof Nordrhein-Westfalen, Ergebnisbericht 2007; Rechnungshof Schleswig-Holstein, Ergebnisbericht 2008.

55 Vgl. Franz-Otto Gilles, a.a.O. (Fn. 48), S. 236; Christopher Pollitt / Hilkka Summa, a.a.O. (Fn. 40), S. 313. 